\title{
Polimorfismos asociados a disfunción endotelial y a un estado protrombótico en jóvenes mexicanos con infarto cerebral
}

\author{
Irma Isordia-Salas, ${ }^{1}$ Rosa María Jiménez-Alvarado, ${ }^{2}$ Megan Carolina Cerda-Mancillas, ${ }^{1}$ \\ José Antonio Alvarado-Moreno, ${ }^{1}$ Jesús Hernández-Juárez, ${ }^{1}$ David Santiago-Germán, ${ }^{3}$ \\ Alfredo Leaños-Miranda ${ }^{4}$ y Abraham Majluf-Cruz ${ }^{1}$ \\ 'Unidad de Investigación Médica en Trombosis, Hemostasia y Aterogénesis, Hospital General Regional No 1. Dr. Carlos Mac Gregor Sánchez \\ Navarro, Instituto Mexicano del Seguro Social (IMSS); ' Instituto de Seguridad y Servicio Social para los Trabajadores del Estado; ${ }^{3}$ Servicio de \\ Urgencias, Hospital General Regional No 1. Dr. Carlos Mac Gregor Sánchez Navarro, IMSS; ${ }^{4}$ Unidad de Investigación Médica en Medicina \\ Reproductiva, Unidad Médica de Alta Especialidad Hospital de Gineco-Obstetricia No. 4, IMSS. Ciudad de México, México
}

\section{Resumen}

Objetivo: Examinar la contribución de los polimorfismos G20210A, G1691A y G10976A en los factores de coagulación Fll, FV y FVII respectivamente; Glu298Asp y C677T en la óxido nítrico sintasa endotelial y 5,10 metilentetrahidrofolato reductasa, en población joven mexicana con infarto cerebral (IC). Método: Se incluyeron 224 pacientes $\leq 45$ años de edad con diagnóstico de IC y 224 controles pareados por edad y sexo, de 2006 a 2014. Los polimorfismos fueron determinados por la técnica de reacción en cadena de la polimerasa-polimorfismos de longitud de fragmentos de restricción. Resultados: Identificamos una diferencia significativa en la distribución genotípica de los polimorfismos Glu298Asp $(p=0.001)$ y C677T ( $p=0.01)$ entre el grupo de pacientes con IC y el control. La distribución genotípica de los polimorfismos FII G20210A, FV G1691A y FVII G10976A fue similar entre ambos grupos. Se identificaron como factores independientes de IC los polimorfismos Glu298Asp y C677T, el tabaquismo, la hipertensión y el antecedente de familiar de enfermedad trombótica. Conclusiones: Los polimorfismos Glu298Asp y C677T, pero no FII G20210A, FV G1691A y FVII G10976A, se asociaron con IC. Nuestros resultados sugieren que la disfunción endotelial en interacción sinérgica con otros factores de riesgo, como tabaquismo e hipertensión, contribuye al IC en individuos jóvenes.

PALABRAS CLAVE: Infarto cerebral, FII. FV. FVII. eNOS. 5,10 MTHFR.

\section{Abstract}

Objective: To examine the contribution the polymorphisms G20210A, G1691A and G10976A in the coagulation factors Fll, FV, FVII, respectively; Glu298Asp and C677T in eNOS and 5,10 MTHFR in young Mexican population with cerebral infarction (CI). Methods: 224 patients $\leq 45$ years of age with $\mathrm{Cl}$ and 224 controls matched by age and gender were recruited from 2006 and 2014. The polymorphisms were determined by polymerase chain reaction-restriction fragment length polymorphism. Results: We identified a significant difference in the genotype distribution of Glu298Asp $(p=0.001)$ and C677T $(p=0.01)$ polymorphisms between Cl patients and control groups. The genotype distribution in the FII G20210A, FV G1691A and FVII G10976A polymorphisms were similar. There were independent factors for ischemic stroke: Glu298Asp and C677T polymorphisms, smoking; hypertension, and familial history of thrombotic disease. Conclusions: The Glu298Asp and C677T, but not FII G20210A, FV G1691A and FVII G10976A polymorphisms were associated with Cl. Our results suggest that endothelial dysfunction and the synergist interaction with other factors such as smoking and hypertension contribute to $\mathrm{Cl}$ in young individuals.

KEY WORDS: Stroke. FII. FV. FVII. eNOS. 5,10 MTHFR.

Correspondencia:

Irma Isordia-Salas

E-mail: irmaiso2016@gmail.com
Fecha de recepción: 16-08-2018

Fecha de aceptación: 20-08-2018

DOI: 10.24875/GMM.18004574
Gaceta Med Mex. 2018;154(Suppl 2): S15-S21

Disponible en PubMed www.gacetamedicademexico.com 


\section{Introducción}

El infarto cerebral (IC) representa la primera causa de discapacidad y la segunda causa de muerte en el mundo. ${ }^{1}$ En nuestro país ocupa la tercera causa de mortali$\mathrm{dad}^{2}$ por lo que es un importante problema de salud pública. El IC en individuos con $\leq 45$ años se presenta en un 3-5\% del total de los casos, en los cuales en más de la mitad no se identifica la etiología. ${ }^{3}$ Es una enfermedad multigénica y multicausal. Estudios clínicos y epidemiológicos han demostrado que los factores de riesgo genéticos tienen una participación crucial en el desarrollo de trombosis en pacientes jóvenes. ${ }^{4}$ Actualmente se reconocen diversos polimorfismos en genes candidatos que codifican proteínas del sistema hemostático, como los factores de coagulación, y también asociados a disfunción endotelial, que se han propuesto como factores de riesgo para el desarrollo de trombosis. ${ }^{5}$

El polimorfismo G20210A en el gen del factor de la coagulación II (FII), o protrombina, está localizado en el cromosoma 11 y se debe a la sustitución del nucleótido guanina en la posición 20210 por adenina, el cual se ha asociado con un incremento en la generación de protrombina, favoreciendo un estado protrombótico. ${ }^{6}$ En 1994, Bertina, et al. ${ }^{7}$ describieron una variante en la secuencia del gen del factor de la coagulación $\mathrm{V}(\mathrm{FV})$ que consiste en el cambio de una base de guanina por una de adenina en la posición 1691 (G1691A), que se traduce en un cambio del aminoácido arginina por glicina en la posición 506 (denominado FV Leiden), causante de la resistencia de la proteína $\mathrm{C}$ activada. Estudios previos han demostrado la asociación del FV Leiden con el riesgo de enfermedad trombótica. También se ha investigado dicha variante como posible factor de riesgo para IC. ${ }^{8}$ Previamente, Kumar, et al..$^{9}$ demostraron, en un estudio de casos y controles, una asociación entre el polimorfismo G1691A y el incremento en el riesgo para IC.

El gen del FVII, localizado en el cromosoma 13q34, es un factor dependiente de la vitamina $\mathrm{K}$, el cual es esencial para la hemostasia, circula en la sangre en forma de zimógeno y se convierte en una forma activa por FIXa, FXa, FXIla o la trombina. Se ha descrito que las concentraciones plasmáticas disminuidas de FVII se asocian a trombosis e IC, mientras que otros estudios no han corroborado la presencia del polimorfismo G10976A en la incidencia de trombosis cerebral. ${ }^{10}$

La enzima óxido nítrico sintasa endotelial (eNOS) cataliza la síntesis de óxido nítrico a partir de L-arginina; el óxido nítrico regula el tono vascular y previene la enfermedad aterotrombótica al inhibir la activación leucocitaria y plaquetaria, y la proliferación de células musculares lisas. ${ }^{11}$ El cambio de guanina por tinina en la posición 894 en el exón 7 del gen que codifica la enzima eNOS, localizado en el cromosoma 7q35-36, se traduce en la sustitución de glutamina (Glu) por aspargina (Asp) en la posición 298, induciendo disfunción endotelial.12 Otro polimorfismo relacionado con disfunción endotelial es el C677T en el gen que codifica a la enzima 5,10 metilentetrahidrofolato reductasa (5,10 MTHFR), localizado en el cromosoma 1p36.3, resulta en la sustitución de valina (Val) por alanina (Ala), y es el causante de una reducción en la actividad de la enzima. ${ }^{13}$ Dicho polimorfismo se ha identificado como un factor de riesgo para IC.12

El objetivo del presente estudio es identificar la asociación y la interacción de los polimorfismos en los factores de coagulación FII G20210A, FV G1691A, FVII G10976A, eNOS G298Asp y 5,10 MTHFR C677T con la presencia de IC en sujetos jóvenes mexicanos.

\section{Método}

En el período comprendido de 2006 a 2014 se reclutaron de manera consecutiva 224 pacientes $\leq 45$ años con diagnóstico de IC. Dicho diagnóstico se estableció en los pacientes con déficit neurológico focal agudo con duración mayor de 24 horas confirmado por tomografía computarizada o resonancia magnética. Se realizaron también ecocardiograma transtorácico y transesofágico, resonancia magnética cardíaca, angiorresonancia y ultrasonido Doppler carotídeo y vertebral para descartar etiologías embólicas y disección arterial cervical. No se incluyeron pacientes con hemorragia cerebral. Posterior a 12 horas de ayuno se tomaron muestras de sangre periférica para determinar las concentraciones de glucosa, colesterol, triglicéridos y colesterol ligado a lipoproteínas de alta densidad y de baja densidad. Se evaluaron los valores de antitrombina, proteína $C$ y proteína $S$ (Diagnostica Stago), así como de anticoagulante lúpico (test DVV, confirmación DVV; American Diagnostic, USA), así como la resistencia a la proteína $C$ activada (normal $>2.0$, Coatest + APC Resistance V-S; Chromogenix, Suecia) y los anticuerpos anticardiolipinas (normal $<10 \mathrm{U}$; Sanofi Diagnostics Pasteur, Francia).

Los individuos con uno o más marcadores de trombofilia se excluyeron del estudio. El grupo control consistió en 224 sujetos sin antecedentes de enfermedad cerebrovascular. Se registraron la edad, el sexo, el uso de anticonceptivos orales, el antecedente de eventos trombóticos y el uso de drogas. Se indagó acerca de otros factores de riesgo para enfermedad aterotrombótica. 
Se incluyeron sujetos con una edad $\leq 45$ años para minimizar el efecto a largo plazo de la influencia ambiental sobre la enfermedad. El diagnóstico de hipertensión arterial se consideró en los sujetos que cumplían los criterios de la Sociedad Europea de Cardiología o que recibían tratamiento antihipertensivo. El antecedente familiar de enfermedad cardiovascular se definió como muerte súbita o enfermedad cardiovascular en un familiar de primer grado varón < 55 años o mujer < 65 años. Tabaquismo positivo se consideró en fumadores activos o que lo hubieran suspendido en los últimos 12 meses. Dislipidemia se consideró en caso de colesterol total $>200 \mathrm{mg} / \mathrm{dl}$ o tratamiento hipolipidemiante. El diagnóstico de diabetes se estableció en pacientes con glucemia en ayuno $>126 \mathrm{mg} / \mathrm{dl}$ o si contaban con el diagnóstico previamente. Los polimorfismos G20210A, G1691A, G10976A, Glu298Asp y C677T de los genes que codifican a FII, FV y FVII, y las enzimas eNOS y 5,10 MTHFR, respectivamente, se determinaron en todos los participantes.

El protocolo de estudio se revisó y aprobó por el Comité de Ética y el Consejo de Investigación Médica del Instituto Mexicano del Seguro Social conforme a los lineamientos de la Declaración de Helsinki de 1975 de la Asociación Médica Mundial, sobre los Principios Éticos para la Investigación Médica que involucra sujetos humanos, adoptada por la 18. ${ }^{a}$ Asamblea Médica Mundial en Helsinki (Finlandia) en 1964, modificada en Tokio (Japón) en 1975 y revisada en Fortaleza (Brasil) en 2013. Se obtuvo el consentimiento informado de todos los participantes previo a su inclusión.

\section{Extracción de ácido desoxirrribonucleico y genotipificación}

El ácido desoxirribonucleico (ADN) genómico se extrajo de muestras de sangre periférica mediante técnicas estandarizadas (QIAamp DNA Blood Mini Kit, Qiagen $\mathrm{GmbH}$, Hilden Alemania).

La amplificación se realizó con 35 ciclos de $1 \mathrm{mi}$ nuto a $95{ }^{\circ} \mathrm{C}, 1$ minuto a $60{ }^{\circ} \mathrm{C}$ y 1 minuto a $72{ }^{\circ} \mathrm{C}$, con una desnaturalización inicial de 5 minutos a 95 ${ }^{\circ} \mathrm{C}$ y una extensión final de 90 segundos a $72{ }^{\circ} \mathrm{C}$. El análisis de polimorfismos de longitud de fragmentos de restricción se realizó con la enzima de restricción Hind III $5 \mathrm{U}$ para el polimorfismo de la protrombina, la Mn1l para el factor de Leiden y la Mspl para el polimorfismo del FVII.

El polimorfismo Glu298Asp del gen de la eNOS requirió una desnaturalización a $94^{\circ} \mathrm{C}$ por 30 segundos, alineación a $60{ }^{\circ} \mathrm{C}$ por 30 segundos y extensión a 72 ${ }^{\circ} \mathrm{C}$ por 30 segundos, por 30 ciclos. Se utilizó la enzima
Mbo I y se obtuvieron dos fragmentos de 119 y $87 \mathrm{pb}$ para el alelo Asp298 y de 206 pb para el Glu298.

El polimorfismo C677T del gen de la 5,10 MTHFR ameritó una desnaturalización a $94{ }^{\circ} \mathrm{C}$ por 20 segundos, alineación a $62{ }^{\circ} \mathrm{C}$ por 20 segundos y extensión a $72{ }^{\circ} \mathrm{C}$ por 20 segundos, por 30 ciclos. Se utilizó la enzima Hinf I y se obtuvo una banda de 175 pb para el alelo T y de 198 pb para el alelo C.

Los fragmentos de ADN se separaron por electroforesis en gel de agarosa al 3\% (BIO-RAD Laboratories, Hercules, California, EE.UU.) y se visualizaron usando bromuro de etidio. Todas las muestras se procesaron por duplicado.

\section{Análisis estadístico}

Las variables continuas se muestran con medias y desviación estándar, y los datos categóricos se expresan con porcentajes. La significancia estadística entre variables continuas se determinó con prueba t de Student. La diferencia entre variables categóricas se evaluó con prueba de ji al cuadrado. Las odds ratio ajustadas se calcularon con análisis multivariado de regresión logística para Ios polimorfismos G20210A, G1691A y G10976A para FII, FV y FVII, respectivamente; Glu298Asp y C677T para eNOS y 5,10 MTHFR, respectivamente; y factores tradicionales de riesgo para IC. Un valor de $p<0.05$ se consideró estadísticamente significativo. Todos los análisis se realizaron utilizando el programa estadístico SPSS (Statistical Package for the Social Sciences) versión 18.

\section{Resultados}

Las características generales de los pacientes y de los controles se muestran en la tabla 1. Todos los participantes tenían ancestros mexicanos. La edad de los pacientes con IC era de $\leq 45$ años. La media de edad para el grupo de pacientes fue de $33.71 \pm 4.6$, mientras que en el grupo control fue de $32.2 \pm 6.5$ años. No hubo diferencia respecto a sexo entre ambos grupos $(p=0.65)$. Debido a que fue un estudio pareado por edad y sexo no encontramos diferencias en dichas variables. Los individuos con IC tuvieron mayor prevalencia de factores tradicionales de riesgo cardiovascular para enfermedad aterotrombótica. Se observó mayor frecuencia de hipertensión en el grupo de pacientes (15.6 vs. 5.3\%), tabaquismo (32.1 vs. $18.7 \%$ ) y antecedente familiar de enfermedad cardiovascular (25.9 vs. 11.2\%). No se encontró diferencia significativa entre los grupos en cuanto a dislipidemia $(p=0.40)$ y diabetes $(p=0.39)$.

La tabla 2 muestra la distribución genotípica de los polimorfismos G20210A, G1691A y G10976A de FII, 
Tabla 1. Características clínicas y demográficas de los pacientes con infarto cerebral y de los sujetos controles

\begin{tabular}{|c|c|c|c|}
\hline & $\begin{array}{l}\text { Pacientes } \\
(\mathrm{n}=224)\end{array}$ & $\begin{array}{l}\text { Controles } \\
(n=224)\end{array}$ & $\mathrm{p}^{*}$ \\
\hline Edad, años (media $\pm \mathrm{DE}$ ) & $33.7 \pm 4.6$ & $32.2 \pm 6.5$ & NS \\
\hline Sexo femenino, $\mathrm{n}(\%)$ & $136(60.7)$ & $136(60.7)$ & NS \\
\hline Sexo masculino, $n(\%)$ & $88(39.3)$ & $88(39.3)$ & \\
\hline Diabetes mellitus, $\mathrm{n}(\%)$ & $21(9.4)$ & $16(7.1)$ & 0.39 \\
\hline Hipertensión, n (\%) & $35(15.6)$ & $12(5.3)$ & 0.001 \\
\hline Tabaquismo activo, n (\%) & $72(32.1)$ & $42(18.7)$ & 0.001 \\
\hline Dislipidemia, n (\%) & $104(46.4)$ & $97(43.3)$ & 0.40 \\
\hline Antecedente familiar de EAT, $\mathrm{n}(\%)$ & $58(25.9)$ & $25(11.2)$ & 0.001 \\
\hline
\end{tabular}

FV y FVII, respectivamente, y Glu298Asp y C677T de eNOS y 5,10 MTHFR, respectivamente, en los grupos de casos y controles.

En la tabla 3 se muestra el análisis multivariado de regresión logística considerando la presencia de IC como variable dependiente. Se identificaron cinco factores de riesgo independientes asociados con IC: los polimorfismos Glu298Asp ( $p=0.01$ ) y C677T ( $p=0.001)$, el tabaquismo $(p=0.01)$, la hipertensión $(p=0.02)$ y el antecedente familiar de enfermedad trombótica $(p=0.03)$.

\section{Discusión}

El IC constituye una enfermedad compleja y heterogénea, de múltiples etiologías con manifestaciones clínicas relevantes. Mientras los factores de riesgo tradicionales, como diabetes, tabaquismo, hipertensión y dislipidemia, son considerados más importantes que los factores hereditarios, diversos estudios de casos y controles, así como metaanálisis, han demostrado una fuerte contribución de factores y de antecedentes genéticos que influyen en la susceptibilidad a sufrir un IC. ${ }^{14}$

Estudios previos han demostrado que el polimorfismo G20210A de la protrombina se asocia con un incremento en la concentración de la proteína, y se ha descrito una frecuencia del 3-5.2\% en población europea. ${ }^{15}$ Si bien los estudios han demostrado la asociación de este polimorfismo G20210A como factor de riesgo para trombosis, ${ }^{16,17}$ en el presente estudio no identificamos la presencia de esta variante genética en una muestra de 224 jóvenes $\leq 45$ años con diagnóstico de IC, ni tampoco en los sujetos que se incluyeron en el grupo control.
Tabla 2. Distribución genotípica y frecuencia alélica de los polimorfismos G20210A, G1691A, G10976A, Glu298Asp y C677T en los genes de FII, FV, FVII, eNOS y 5,10 MTHFR, respectivamente, en 224 pacientes con IC $\leq 45$ años y sus controles

\begin{tabular}{|c|c|c|c|c|}
\hline Locus & Genotipo & $\begin{array}{c}\text { Casos } \\
(n=224)\end{array}$ & $\begin{array}{l}\text { Controles } \\
(n=224)\end{array}$ & $\mathbf{p}^{*}$ \\
\hline & $\mathrm{G} / \mathrm{G}$ & $224(100.0)$ & $224(100.0)$ & NS \\
\hline \multirow[t]{3}{*}{ G20210A FII } & $\mathrm{G} / \mathrm{A}$ & $0(0.0)$ & $0(0.0)$ & \\
\hline & $A / A$ & $0(0.0)$ & $0(0.0)$ & \\
\hline & $\mathrm{G} / \mathrm{G}$ & $224(100.0)$ & $224(100.0)$ & NS \\
\hline \multirow[t]{3}{*}{ G1691A FV } & $\mathrm{G} / \mathrm{A}$ & $0(0.0)$ & $0(0.0)$ & \\
\hline & $\mathrm{A} / \mathrm{A}$ & $0(0.0)$ & $0(0.0)$ & \\
\hline & $\mathrm{G} / \mathrm{G}$ & $182(81.25)$ & $180(80.4)$ & 0.58 \\
\hline \multirow[t]{3}{*}{ G10976A FVII } & $\mathrm{G} / \mathrm{A}$ & $18(18.3)$ & $40(17.8)$ & \\
\hline & $\mathrm{A} / \mathrm{A}$ & $1(0.45)$ & $4(3.8)$ & \\
\hline & Glu/G & $118(52.7)$ & $159(71.0)$ & 0.001 \\
\hline \multirow[t]{3}{*}{ Glu298Asp eNOS } & Glu/Asp & $93(41.5)$ & $58(25.9)$ & \\
\hline & Asp/Asp & $13(5.8)$ & $7(3.1)$ & \\
\hline & $\mathrm{C} / \mathrm{C}$ & $42(18.7)$ & $74(33.0)$ & 0.01 \\
\hline \multirow[t]{2}{*}{ C677T 5,10 MTHFR } & $\mathrm{C} / \mathrm{T}$ & $135(60.3)$ & $102(45.5)$ & \\
\hline & $T / T$ & $47(21.0)$ & $48(21.5)$ & \\
\hline
\end{tabular}

FII: factor II o protrombina; FV: factor V o factor V de Leiden; FVII: factor VII eNOS: enzima óxido nítrico sintasa endotelial; 5,10 MTHFR: 5,10 metilentetrahidrofolato reductasa; NS: no significativa.

*Prueba de ji al cuadrado.

Tabla 3. Análisis multivariado de regresión logística utilizando el infarto cerebral como variable dependiente

\begin{tabular}{lccc}
\hline Factor de riesgo & OR & IC95\% & $\mathbf{p}^{*}$ \\
\hline Glu298Asp & 1.6 & $(1.0-3.9)$ & 0.001 \\
C677T & 2.6 & $(1.2-4.6)$ & 0.01 \\
Hipertensión & 2.40 & $(1.4-4.75)$ & 0.02 \\
Tabaquismo & 2.71 & $(1.1-4.37)$ & 0.01 \\
Antecedente familiar de EAT & 1.68 & $(1.0-2.79)$ & 0.03 \\
\hline $\begin{array}{l}\text { EAT: enfermedad aterotrombótica; IC: intervalo de confianza; OR: odds ratio ajustada. } \\
\text { *Prueba de ji al cuadrado en regresión logística múltiple. }\end{array}$ & &
\end{tabular}

Nuestros resultados son similares a los reportados por Saadatnia, et al., ${ }^{18}$ quienes no observaron la presencia del alelo A polimórfico en forma heterocigota GA ni homocigota AA en población iraní. Además, They-They, et al. ${ }^{19}$ demostraron que el genotipo G20210A no presenta una asociación estadísticamente significativa entre la población estudiada y el grupo control, y Bolaman, et al. ${ }^{20}$ reportan en su estudio en población turca que el polimorfismo G20210A no fue identificado. $^{20}$ En un estudio similar de casos y 
controles con una muestra de 100 pacientes, Lopaciuk, et al. ${ }^{21}$ demostraron que dicho polimorfismo no se asocia a IC en sujetos jóvenes en población polaca. En forma contraria, existen reportes de estudios en los cuales se ha demostrado un incremento en el porcentaje obtenido del polimorfismo G20210A en el grupo de pacientes comparados con los controles, como fue descrito por Eterovic, et al..$^{22}$ en población croata.

Diversos estudios han demostrado que el polimorfismo G1691A en el FV, también denominado factor $\mathrm{V}$ de Leiden, representa un factor de riesgo para trombosis venosa profunda en población latina ${ }^{23} \mathrm{y}$ para IC en pacientes pediátricos. ${ }^{24}$ Sin embargo, la participación de dicho polimorfismo en la enfermedad aterotrombótica en la edad adulta aún es controversial. ${ }^{25}$ En este estudio, el polimorfismo G1691A no se presentó en forma heterocigota GA ni homocigota AA en los casos ni en los controles. En forma contraria, Hamedani, et al. ${ }^{26}$ demostraron una asociación entre el FV y el IC en sujetos menores de 50 años.

Además, se ha evidenciado que un incremento en la concentración de FVII se asocia con un riesgo mayor para enfermedades aterotrombóticas como el infarto agudo de miocardio y el infarto cerebral. ${ }^{27}$ Estudios previos han demostrado que el polimorfismo G10976A es causa de hasta un 50\% de la concentración plasmática de $\mathrm{FVII}{ }^{28}$ y se asocia con una disminución del $20-25 \%$ de dicha proteína. En estudios previos, el polimorfismo G10976A del FVII ha sido demostrado como un factor "protector" para el desarrollo de enfermedades aterotrombóticas como el infarto agudo de miocardio y el IC, ${ }^{29,30}$ probablemente debido a la baja concentración de FVII circulante. En el presente estudio, el polimorfismo G10976A del FVII no estuvo asociado con el IC. Nuestros resultados son acordes a los publicados por Maguire, et al. ${ }^{31}$ en un metaanálisis en el que se incluyeron seis estudios con un total de 1537 casos y 3133 controles, quienes demuestran una fuerte evidencia de que dicho polimorfismo no representa un factor de riesgo para el IC. En el estudio realizado por Haeusler, et al. ${ }^{32}$ se evaluó a 29 pacientes jóvenes y se encontró el genotipo GG en un $3.4 \%$ y el genotipo $A A$ en un $3.4 \%$, mientras que en el grupo control el porcentaje obtenido fue del $20.2 \%$ para el genotipo GA y del $1.8 \%$ para el genotipo AA. Los resultados anteriores difieren de lo reportado en el presente estudio, en el cual obtuvimos un $18.30 \%$ para el genotipo GG y menos del $1 \%(0.45 \%)$ para el genotipo AA, y para el grupo control los resultados fueron similares, con un $17.8 \%$ para los heterocigotos GA y un $1.8 \%$ para los sujetos con doble alelo $A(A A)$. Las diferencias poblacionales obtenidas en la distribución de un determinado genotipo podrían ser importantes en la posible influencia de dicho gen en la enfermedad, así como su interacción con los factores de riesgo tradicionales. También demostramos que el polimorfismo G10976A del FVII no se asocia a infarto agudo de miocardio en sujetos jóvenes mexicanos, ${ }^{33}$ lo cual se ha descrito en población japonesa, ${ }^{34}$ y ello podría confirmar que dicha variante genética favorece el desarrollo de trombosis venosa más que en el territorio arterial.

Como ha sido demostrado por estudios de asociación de genes, diversos polimorfismos son particularmente importantes en la predisposición a sufrir un IC. La identificación de nuevos marcadores genéticos asociados a un incremento en el riesgo de IC es esencial para la predicción del riesgo, la prevención y el tratamiento. ${ }^{35}$

En el presente estudio, el polimorfismo Glu298Asp del gen de la eNOS se asoció con la presencia de IC en sujetos jóvenes $\leq 45$ años mexicanos (genotipo $p=0.001$ y frecuencia alélica $p=0.001$, respectivamente). Nuestros resultados son acordes con los publicados por Saidi, et al., ${ }^{36}$ quienes reportaron que la variación genética Glu298Asp representa un factor independiente para IC. Además, en un metaanálisis que incluyó 6733 casos y 7305 controles se observó una asociación estadísticamente significativa del polimorfismo Glu298Asp con la presencia de IC. ${ }^{37}$

Diversos estudios han demostrado que una deficiencia de óxido nítrico favorece el desarrollo y la progresión del proceso ateroscleroso. Además, el óxido nítrico es un potente vasodilatador y regula la presión arterial y el flujo sanguíneo. También inhibe la adhesión y la agregación plaquetarias, la adhesión leucocitaria y la proliferación y la migración de las células musculares lisas. La deficiencia de óxido nítrico se considera un factor importante para disfunción endotelial. ${ }^{38}$

En años recientes se ha demostrado que la hiperhomocisteinemia se asocia a un incremento en el riesgo de enfermedades trombóticas. La homocisteína es un aminoácido formado durante el metabolismo de la metionina; el incremento en sus concentraciones plasmáticas se ha asociado a disfunción del metabolismo cerebral, y es considerado un factor de riesgo independiente de IC. ${ }^{39}$ En el presente estudio, el genotipo homocigoto TT y heterocigoto CT del polimorfismo C677T en el gen de la 5,10 MTHFR se asoció con la presencia de IC en sujetos jóvenes $\leq 45$ años mexicanos $(p=0.01)$. Nuestros resultados coinciden con los publicados por Jiang, et al.., ${ }^{40}$ quienes demostraron una asociación entre el polimorfismo C677T y el IC.40 En contraste, Ranellou, et al. ${ }^{41}$ no observaron diferencia significativa en la frecuencia alélica del polimorfismo C677T en pacientes < 50 años griegos con IC en 
comparación con sujetos controles. Además, identificamos un incremento significativo $(p<0.001)$ en las concentraciones séricas de homocisteína posterior a una carga oral de metionina en un grupo de pacientes jóvenes con IC en comparación con sujetos controles. Sin embargo, nuestros resultados son contrarios a los publicados por $\mathrm{Li}$, et al., ${ }^{42}$ quienes demostraron de igual forma una asociación del polimorfismo C677T con un incremento en el riesgo para IC, pero también que dicha variante se asocia con un incremento en la concentración de homocisteína en el mismo grupo de pacientes con una edad promedio de 62 años.

La inconsistencia de los resultados entre los diferentes estudios sugiere que los pacientes con IC poseen un genotipo cerebrovascular complejo. Es necesaria una cuidadosa estratificación etiológica de los pacientes, estudios epidemiológicos en poblaciones con mayor número de pacientes y controles, una detallada evaluación funcional de la variante genética, y la validación de los resultados en diferentes grupos étnicos, para determinar la exacta participación de estos genes en el desarrollo de IC. ${ }^{43}$ Como ha sido demostrado por estudios de asociación de genes, diversos polimorfismos son particularmente importantes en la predisposición al IC. La identificación de nuevos marcadores genéticos asociados a un incremento en el riesgo de IC es esencial para la predicción del riesgo, la prevención y el tratamiento.

Existen diversos estudios en los que se demuestra una interacción de diversas variantes genéticas y el IC, como se ha demostrado en un metaanálisis en el que se incluyeron 490 estudios de casos y controles, con 138592 casos y 159314 controles. La evidencia del estudio concluyó que los polimorfismos C677T del gen de la metiltetrahidrofolato reductasa (MTHFR), Glu298Asp del gen de la eNOS y el M235T del gen de AGT predisponen al IC. ${ }^{44}$ Además, Szolnoki, et al. ${ }^{45}$ demostraron que el polimorfismo Glu298AS o Asp298Asp en combinación con MTHFR/C77T o ACE/DD incrementa el riesgo para IC en población caucásica.

Lo anterior confirma que, en una enfermedad multigénica y multifactorial, la interacción de diversas variantes genéticas, así como la presencia de diversos factores de riesgo modificables, pueden conducir a enfermedades trombóticas de tipo arterial en edades tempranas de la vida.

De manera similar a lo descrito en otros estudios en población joven, la hipertensión y el tabaquismo se asociaron con IC, persistiendo como factores de riesgo independientes después del ajuste por otras variables. La diabetes mellitus y la dislipidemia no se relacionaron con IC en nuestra población, de modo semejante a lo descrito previamente. ${ }^{46}$ En contraste, los estudios realizados en población de mayor edad con IC han encontrado que la diabetes mellitus y la dislipidemia son factores de riesgo para IC.46 Basándonos en estos hechos, consideramos que los factores de riesgo aterotrombótico pueden no tener la misma influencia en el IC en pacientes jóvenes que en los de mayor edad, y que la interacción de esos factores y variantes genéticas específicas difiere para cada grupo de edad. ${ }^{47}$

El IC es una enfermedad con un espectro clínico y etiológico muy heterogéneo, en la cual la prevalencia de factores de riesgo tradicionales, genéticos y ambientales varía de una población a otra, además de tener diferente influencia en las distintas etapas de la vida, lo cual probablemente determine el desarrollo y la edad de presentación del IC, explicando en parte la inconsistencia de los resultados.

Algunas fortalezas del estudio son: 1) los grupos de estudio fueron pareados por edad y sexo; 2) la edad se limitó a $\leq 45$ años para minimizar el efecto del tiempo de exposición a los factores de riesgo tradicionales; y 3) se analizaron cinco polimorfismos en cinco genes candidatos del sistema hemostático en FII, FV, FVII, eNOS y 5,10 MTHFR en cada individuo. Una limitante es que no se determinó la concentración plasmática de FII, FV y FVII.

En conclusión, los polimorfismos Glu298Asp y C677T, pero no FIIG20210A, FV G1691A y FVII G1097A, se asocian a un incremento en el riesgo de IC en sujetos jóvenes mexicanos. Se identificaron como factores independientes para IC los polimorfismos Glu298Asp y C677T, el tabaquismo, la hipertensión y el antecedente familiar de enfermedad aterotrombótica. Nuestros resultados demuestran que probablemente el mecanismo para IC en individuos jóvenes se deba a disfunción endotelial y a la interacción sinérgica con otros factores de riesgo modificables, como el tabaquismo y la hipertensión. Estos factores quizá contribuyan al IC a edad temprana, mientras que los polimorfismos en FII, FV y FVII probablemente tengan más influencia en la trombosis venosa, como ha sido demostrado en diversas poblaciones.

\section{Agradecimientos}

Esta investigación recibió financiamiento del Fondo de Investigación en Salud IMSS (FIS/IMSS/PROT/ PRIO/13/023) y del Fondo Sectorial de Investigación 
en Salud y Seguridad Social (SS-IMSS-/ISSSTE/CONACYT. No. 261887).

\section{Conflictos de intereses}

\author{
Los autores declaran no tener ningún conflicto de
} intereses.

\section{Bibliografía}

1. American Heart Association. Heart disease and stroke statistics 2009 update: a report from the American Heart Association Statistics Committee and Stroke Statistics Subcommittee. Circulation. 2009:119:121-81.

2. Instituto Nacional de Estadística, Geografía e Informática. Estadísticas de mortalidad (México). (Consultado el 18/2/2018.) Disponible en: http:// www.beta.inegi.org.mx/proyectos/registros/vitales/mortalidad/

3. Putaala J, Metso AJ, Metso TM, Konkola N, Kraemer Y, Haapaniemi E, et al. Analysis of 1008 consecutive patients aged from 15 to 49 with first event ischemic stroke: the Helsinki young stroke registry. Stroke. 2009;40:1195-203.

4. Madonna P, De Stefano V, Coppola A, Cirillo F, Cerbone A, Orefice G, et al. Hiperhomocysteinemia and other inherited prothrombotic conditions in young adults with a history of ischemic stroke. Stroke. 2002;33:51-6.

5. Pruissen DM, Kappelle LJ, Rosendaal FR, Algra A. Genetic association studies in ischaemic stroke: replication failure and prospects. Cerebrovasc Dis. 2009;27:290-4.

6. Jiang B, Ryan KA, Hamedani A, Cheng Y, Sparks MJ, Koontz D, et al. Prothrombin G20210A mutation is associated with young-onset stroke: the genetics of early-onset stroke study and meta-analysis. Stroke. 2014;45:961-7.

7. Bertina RM, Koeleman BPC, Koster T, Rosendaal FR, Dirven RJ, de Ronde $\mathrm{H}$. Mutation in blood coagulation factor $\mathrm{V}$ associated with resistance to activated protein C. Nature. 1994;369:64-7.

8. Ridker PM, Hennekenns CH, Lindpainter K, Stamfer MJ, Eisenberg PR. Mutation in gene coding for coagulation factor $\mathrm{V}$ and the risk of myocardial infarction, stroke, and venous thrombosis in apparently healthy men. N Engl J Med. 1995;33:912-7.

9. Kumar A, Misra S, Sagar R, Kumar P, Yadav AK, Talwar P, et al. Relationship between factor $\mathrm{V}$ Leiden gene variant and risk of ischemic stroke: a case-control study. Ann Indian Acad Neurol. 2017;20:284-8.

10. Evangelista FC, Rios DR, Ribeiro DD, Carvalho MG, Dusse LM, Fernandes AP et al. Lack of association between potential prothrombotic genetic risk factors and arterial and venous thrombosis. Genet Mol Res. 2015;14:9585-94.

11. Lloyd-Jones DM, Bloch KD. The vascular biology of nitric oxide and its role in atherogenesis. Annu Rev Med. 1996:47:365-75.

12. Marsden PA, Heng HH, Scherer SW, Stewart RJ, Hall AV, Shi XM, et al. Structure and chromosomal localization of the human constitutive endothelial nitric oxide synthase gene. J Biol Chem. 1993;268:17478-88

13. Frosst $P$, Blom HJ, Milos R, Goyette P, Sheppard CA, Matthews RG, et al A candidate genetic risk factor for vascular disease: a common mutation in methylenetetrahydrofolate reductase. Nutr Genet. 1995;10:111-3.

14. You RX, McNeil JJ, O'Malley HM, Davis SM, Thrift AG, Donnan GA. Risk factors for stroke due to cerebral infarction in young adults. Stroke. 1997;28:1913-8

15. Poort SW, Rosendaal FR, Reitsma PH, Bertina RM. A common genetic variation in the 3' untraslated region of the prothrombin gene is associated with elevated plasma prothrombin levels and an increase in venous thrombosis. Blood. 1996;88:3698-703.

16. De Stefano V, Chiusolo P, Paciaroni K, Casorelli ID, Rossi E. Prothrombin G20210A mutant genotype is a risk factor for cerebrovascular ischemic disease in young patients. Blood. 1998;91:3562-5.

17. Reuner KH, Ruf A, Grau A, Rickmann H, Stolz E, Jüttler E, et al. Prothrombin gene $\mathrm{G} 20210 \rightarrow A$ transition is a risk factor for cerebral venous thrombosis. Stroke. 1998;29:1765-9.

18. Saadatnia M, Salehi M, Amini G, Seyyed Agha Miri N. The impact of prothrombin (G20210A) gene mutation on stroke in youths. ARYA Atheroscler. 2012;8:9-11.

19. They-They TP, Battas O, Slassi I, Rafai MA, Katumbay DT, Nadifi S. Prothrombin G20210A and factor V Leiden polymorphisms in stroke. J Mol Neurosci. 2012;46:210-6.

20. Bolaman Z, Ozkul A, Kiylioglu N, Kadikoylu G, Erturk A, Batun S, et al. Hereditary thrombophilic factors in stroke due to cerebral infarct. Am Med Sci. 2009:337:11-3.

21. Lopaciuk S, Bykowska K, Kwiecinski H, Mickielewicz A, Czlonkowska A Mendel T, et al. Factor V Leiden, prothrombin gene G20210A variant and methylenetetrahydrofolate reductase C677T genotype in young adults with ischemic stroke. Clin Appl Thromb Hemost. 2001;7:346-50.

22. Eterović D, Titlić M, Culić V, Zadro R, Primorac D. Lower contribution of factor V Leiden or G202104 mutations to ischemic stroke in patients with clinical risk factors: pair-matched case-control study. Clin Appl Thromb Hemost. 2007;13:188-93.

23. Pestana $\mathrm{Cl}$, Torres A, Blanco S, Rojas MJ, Méndez C, López JL, et al. Factor $\mathrm{V}$ Leiden and the risk of venous thrombosis, myocardial infarction, and stroke: a case-control study in Venezuela. Genet Test Mol Biomarkers. 2009;13:537-42.

24. Kenet G, Sadetzki S, Murad H, Martinowitz U, Rosenberg N, Gitel S, et al. Factor V Leiden and antiphospholipid antibodies are significant risk factors for ischemic stroke in children. Stroke. 2000;31:1283-8.

25. Voetsch B, Damasceno BP, Camargo EC, Massaro A, Bacheschi LA, Scaff $M$, et al. Inherited thrombophilia as a risk factor for the development of ischemic stroke in young adults. Thromb Haemost. 2000:83:229-33.

26. Hamedani AG, Cole JW, Mitchell BD, Kittner SJ. Meta-analysis of factor $\mathrm{V}$ Leiden and ischemic stroke in young adults: the importance of case ascertainment. Stroke. 2010;41:1599-603.

27. Green F, Kelleher C, Wilkes H, Temple A, Meade T, Humphries S. A common genetic polymorphism associated with lower coagulation factor VII levels in healthy individuals. Arterioscler Thromb. 1991;1:540-6.

28. Turfan M, Poyraz F, Kaymak AÖ, Ergun MA, Tavil Y, Gursel T, et al. Plasma levels and distribution of gene polymorphisms of factor VII in Turkish population. Clin Appl Thromb Hemost. 2014;20:164-8.

29. Wu AH, Tsongalis GJ. Correlation of polymorphism to coagulation and biochemical risk factors for cardiovascular diseases. Am J Cardiol. 2001;87:1361-6.

30. Lane A, Green F, Scarabin PY, Nicaud V, Bara L, Humphries S, et al. Factor VII Arg/Gln353 polymorphism determines factor VII coagulant activity in patients with myocardial infarction (MI) and control subjects in Belfast and in France but is not a strong indicator of MI risk in the ECTIM study. Atherosclerosis. 1996:119:119-27.

31. Maguire JM, Thakkinstian A, Sturm J, Levi C, Lincz L, Parsons M, et al. Polymorphisms in platelet glycoprotein $1 \mathrm{~b}$ alpha and factor VII and risk of ischemic stroke: a meta-analysis. Stroke. 2008:39:1710-6.

32. Haeusler KG, Herm J, Hoppe B, Kasabov R, Malzahn U, Endres M, et al. Thrombophilia screening in young patients with cryptogenic stroke. Prevalence of gene polymorphisms compared to healthy blood donors and impact on secondary stroke prevention. Hamostaseologie. 2012;32:147-52.

33. Valades-Mejía MG, Domínguez-López ML, Aceves-Chimal JL, Miranda AL, Majluf-Cruz A, Isordia-Salas I. Study of the polymorphism R353Q in the coagulation factor VII gene and the N700S in the thrombospondin-1 gene in young patients with acute myocardial infarction. Cir Cir. 2014;82:595-606.

34. Ogawa M, Abe S, Biro S, Saigo M, Kihara T, Setoyama S, et al. R353Q polymorphism, activated factor VII, and risk of premature myocardial infarction in Japanese men. Circ J. 2004;68:520-5.

35. Miao Y, Liao JK. Potential serum biomarkers in the pathophysiological processes of stroke. Expert Rev Neurother. 2014:14:173-85.

36. Saidi S, Mallat SG, Almawi WY, Mahjoub T. Endothelial nitric oxide synthase Glu298Asp, 4b/a, and -786T>C gene polymorphisms and the risk of ischemic stroke. Acta Neurol Scand. 2010;121:114-9.

37. Kumar A, Misra S, Kumar P, Prasad K, Pandit AK, Chakravarty K, et al. Association between endothelial nitric oxide synthase gene polymorphisms and risk of ischemic stroke: a meta-analysis. Neurol India. 2017;65:22-34.

38. Arnal JF, Dinh-Xuan AT, Pueyo M, Darblade B, Rami J. Endothelium-derived nitric oxide and vascular physiology and pathology. Cell Mol Life Sci. 1999;55:1078-87.

39. Zhao M, Wang $X$, He M, Qin X, Tang G, Huo $Y$, et al. Homocysteine and stroke risk: modifying effect of methylenetetrahydrofolate reductase C677T polymorphism and folic acid intervention. Stroke. 2017;48:1183-90.

40. Jiang S, Li J, Zhang Y, Venners SA, Tang G, Wang Y, et al. Methylenetetrahydrofolate reductase C677T polymorphism, hypertension and risk of stroke: a prospective, nested case-control study. Int J Neurosci. 2017;127:253-60.

41. Ranellou K, Paraskeva A, Kyriazopoulos P, Batistatou A, Evangelou A, El-Aly $\mathrm{M}$, et al. Polymorphisms in prothrombotic genes in young stroke patients in Greece: a case-controlled study. Blood Coagul Fibrinolysis. 2015;26:430-5.

42. Li A, Shi $Y, X u L$, Zhang $Y$, Zhao $H$, Li Q, et al. A possible synergistic effect of MTHFR C677T polymorphism on homocysteine level variations increased risk for ischemic stroke. Medicine (Baltimore). 2017;96:e9300.

43. Maasz A, Melegh B. Three periods of one and a half decade of ischemic stroke susceptibility gene research: lessons we have learned. Genome Med. 2010;2:64

44. Wei LK, Au A, Menon S, Griffiths LR, Kooi CW, Irene L, et al. Polymorphisms of MTHFR, eNOS, ACE, AGT, ApoE, PON1, PDE4D, and ischemic stroke: meta-analysis. J Stroke Cerebrovasc Dis. 2017:26:2482-93.

45. Szolnoki Z, Havasi V, Bene J, Komlósi K, Szöke D, Somogyvári F, et al, Endothelial nitric oxide synthase gene interactions and the risk of ischaemic stroke. Acta Neurol Scand. 2005;111:29-33.

46. Putaala J, Metso AJ, Metso TM, Konkola N, Kraemer Y, Haapaniemi E, et al. Analysis of 1,008 consecutive patients aged from 15 to 49 with first event ischemic stroke: the Helsinki young stroke registry. Stroke. 2009:40:1195-203.

47. Flossman E, Schulz UGR, Rothwell PM. Systematic review of methods and results of studies of the genetic epidemiology of ischemic stroke. Stroke. 2004;35:212-27. 